\title{
Wave functions and leptonic decays of bottom mesons in the relativistic potential model
}

\author{
Hao-Kai Sun ${ }^{*}$ and Mao-Zhi Yang ${ }^{\dagger}$ \\ School of Physics, Nankai University, Tianjin, China
}

(Received 11 March 2019; published 10 May 2019)

\begin{abstract}
We study the wave functions and purely leptonic decays of $b$-flavored mesons (pseudoscalars, vector mesons, and higher excited states that are well established in experiment) in the relativistic potential model based on our previous works. The wave functions are obtained by solving the wave equation including the spin-spin and spin-orbit corrections in the effective potential. The decay constants of $B, B^{*}$, and some of their excited states that have been found in experiment are calculated with these wave functions. Then the branching fractions of the purely leptonic decay modes of these bottom mesons are studied. Our results are well in agreement with experimental data for decay modes that have been measured in experiments. We also provide predictions for some yet unmeasured channels, which are useful for experimental testing in the future.
\end{abstract}

DOI: 10.1103/PhysRevD.99.093002

\section{INTRODUCTION}

Recently, the ATLAS Collaboration updated the branching ratios of $\mathcal{B}\left(B_{s} \rightarrow \mu^{+} \mu^{-}\right)=\left(2.8_{-0.7}^{+0.8}\right) \times 10^{-9}$ and $\mathcal{B}\left(B_{d} \rightarrow \mu^{+} \mu^{-}\right)<2.1 \times 10^{-10}$ with larger collision data samples [1] at the $95 \%$ confidence level. The former decay channel imposes severe constraints on theoretical study, especially for physics beyond the Standard Model (BSM) $[2,3]$. In general, the purely leptonic decays with a final lepton-neutrino pair or lepton-lepton pair are considered as rare decays, which have relatively simpler physics than hadronic decays. Their decay rates are connected straightforwardly with the Cabibbo-Kobayashi-Maskawa (CKM) matrix elements $[4,5]$ and the bound-state properties of the bottom meson.

Compared with our previous work [6], where only decay constants of pseudoscalar $B$ and $B_{s}$ mesons are considered, here we extend our earlier work by including the decay constants and pure leptonic decays of vector and higher excited states of bottom mesons. We upgrade the scenario for treating the energy-momentum conservation for the quark-antiquark inside the bottom mesons. The theoretical results for the leptonic decays are compared with experimental data. For the measured decay modes, our predictions are well consistent with experiment. For the yet

\footnotetext{
*sunhk@mail.nankai.edu.cn

†yangmz@nankai.edu.cn
}

Published by the American Physical Society under the terms of the Creative Commons Attribution 4.0 International license. Further distribution of this work must maintain attribution to the author(s) and the published article's title, journal citation, and DOI. Funded by SCOAP ${ }^{3}$. unmeasured decay modes, our prediction could be useful for experimental testing in the future.

The paper is organized as follows: In Sec. II, we briefly present the theoretical framework for the relativistic potential model, given the solved wave function for the bottom mesons. The formulas to calculate the decay constants and branching ratios are also given here. Section III is devoted to numerical results and discussions. Finally, Sec. IV is for the conclusion and summary.

\section{THEORETICAL FRAMEWORK}

\section{A. Relativistic potential model and bound-state wave functions}

The heavy-light quark-antiquark bound-state systems have been extensively studied with the relativistic potential model in our previous works [6-9]. The bound-state wave functions of mesons can be obtained by solving a Schrödinger-type equation

$$
\left(H_{0}+H^{\prime}\right) \psi(\vec{r})=E \psi(\vec{r}),
$$

where $H_{0}+H^{\prime}$ is the effective Hamiltonian, which can be found in Ref. [9], and $E$ is the meson's energy. The term $H_{0}$ reads

$$
H_{0}=\sqrt{\vec{p}_{1}^{2}+m_{1}^{2}}+\sqrt{\vec{p}_{2}^{2}+m_{2}^{2}}+V(r)
$$

with

$$
V(r)=-\frac{4}{3} \frac{\alpha_{S}(r)}{r}+b r+c,
$$




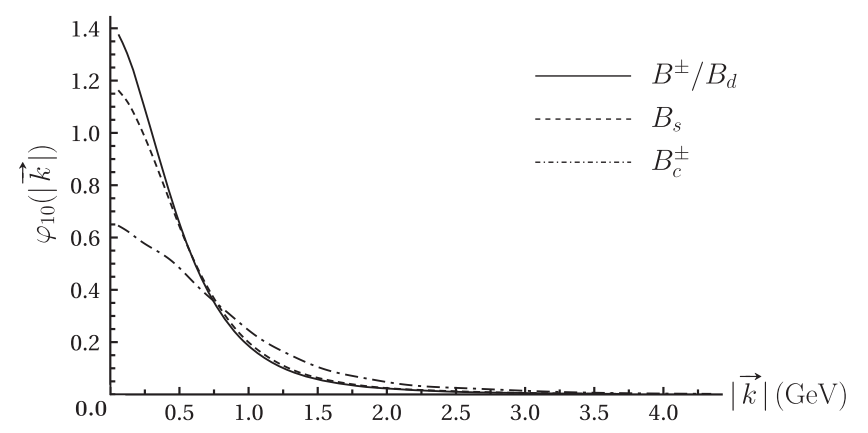

FIG. 1. Radial wave functions of pseudoscalar mesons, normalized by multiplying a constant $Y_{00}=1 / \sqrt{4 \pi}$.

where $V(r)$ is the effective potential for the strong interaction between the quark and antiquark [10-12]. The first term, $-\frac{4}{3} \frac{\alpha_{S}(r)}{r}$ in $V(r)$, originates from the onegluon-exchange diagram for the short-distance contributions, and $b r$ is for confinement effects at a long distance, while $c$ is a phenomenological parameter for this heavylight quark-antiquark system. The other term, $H^{\prime}$, contains spin-spin hyperfine interactions and spin-orbit interactions, which are not given explicitly here (see Ref. [9]).

Using the method described in Ref. [7] and developed in Refs. [8,9], the wave equation in Eq. (1) can be solved numerically. The wave functions in momentum space can be written as

$$
\psi_{n l m}(\vec{k})=\varphi_{n l}(k) Y_{l m}(\hat{k}),
$$

where the subscripts $n l m$ stand for the $n$th radial wave function ( $n=1$ is the lowest), the orbital angular momentum quantum number $l(l=0,1,2, \ldots)$, and the magnetic quantum number $m$ corresponding to $l . \varphi_{n l}(k)$ is the radial wave function, and $Y_{l m}(\hat{k})$ is the spherical harmonics. The normalization condition for the wave function is

$$
\int d k^{3}\left|\psi_{n l m}(\vec{k})\right|^{2}=1
$$

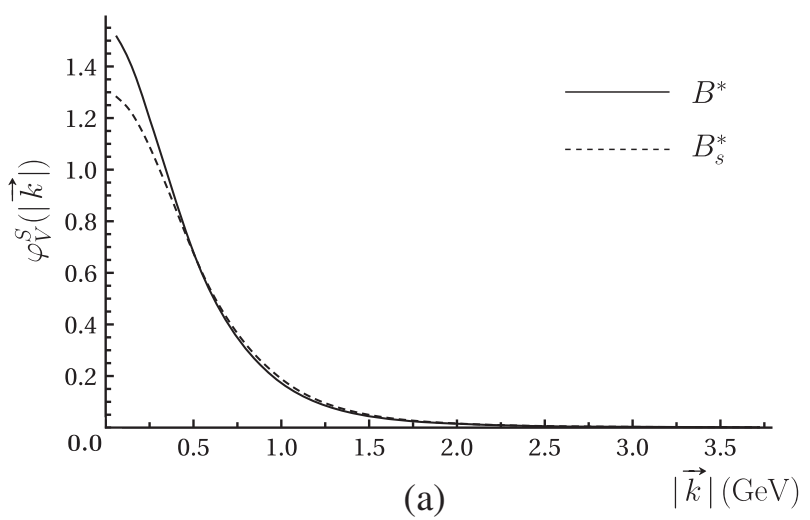

FIG. 2. Radial wave functions of vector mesons (a) for the S-wave parts and (b) for the D-wave parts.
TABLE I. Parameters of fitting functions for pseudoscalar $B$

\begin{tabular}{lcrrc}
\hline \hline Mesons & $a_{1}\left(\mathrm{GeV}^{-3 / 2}\right)$ & $a_{2}\left(\mathrm{GeV}^{-2}\right)$ & $a_{3}\left(\mathrm{GeV}^{-1}\right)$ & $a_{4}$ \\
\hline$B^{ \pm} / B_{d}$ & $1.66_{-0.11}^{+0.07}$ & $-1.07_{-0.16}^{+0.12}$ & $-0.98_{-0.12}^{+0.17}$ & $-0.13_{-0.04}^{+0.02}$ \\
$B_{s}$ & $1.97_{-0.09}^{+0.05}$ & $-1.09_{-0.18}^{+0.12}$ & $-0.69_{-0.10}^{+0.07}$ & $-0.47_{-0.07}^{+0.03}$ \\
$B_{c}$ & $1.04_{-0.03}^{+0.02}$ & $-0.51_{-0.08}^{+0.07}$ & $-0.44_{-0.07}^{+0.04}$ & $-0.43_{-0.04}^{+0.02}$ \\
\hline \hline
\end{tabular}

The details for solving the wave equation can be found in our previous works in Refs. [7-9], which will not be given here for briefness. By solving the wave equation numerically, the wave function can be obtained. In practice, it is convenient to give an analytical form for radial wave functions by fitting the numerical solution. We find that the wave function can be fitted with the following exponential form:

$$
\varphi_{n l}(\vec{k})=a_{1} e^{a_{2}|\vec{k}|^{2}+a_{3}|\vec{k}|+a_{4}} .
$$

Next, we give the obtained results for the parameters $a_{1}-a_{4}$ for each quantum state.

(1) For pseudoscalars $J^{P}=0^{-}$, we ignore the difference between the light quark masses $m_{u}$ and $m_{d}$; therefore, the radial wave functions of $B^{ \pm}$and $B_{d}$ shall be the same. The wave functions of $B^{ \pm} / B_{d}, B_{s}$, and $B_{c}^{ \pm}$ are depicted in Fig. 1. It is noted that the difference between $B^{ \pm} / B_{d}$ and $B_{s}$ is relatively smaller than that between $B^{ \pm} / B_{d}$ and $B_{c}^{ \pm}$, since the mass of the $c$ quark is greatly larger than those of the light quarks. The results for the parameters $a_{1}, a_{2}, a_{3}, a_{4}$ we obtained are listed in Table I.

(2) For vector mesons $J^{P}=1^{-}$, in our model [8,9], they are mixing-states of S-wave $(l=0)$ eigenstate $\left|{ }^{3} S_{1}\right\rangle$ and D-wave $(l=2)$ eigenstate $\left|{ }^{3} D_{1}\right\rangle$. The $\mathrm{S}$-wave and $\mathrm{D}$-wave functions should be given separately. We use $\psi_{V}^{S}(\vec{k})$ to denote the $\mathrm{S}$-wave

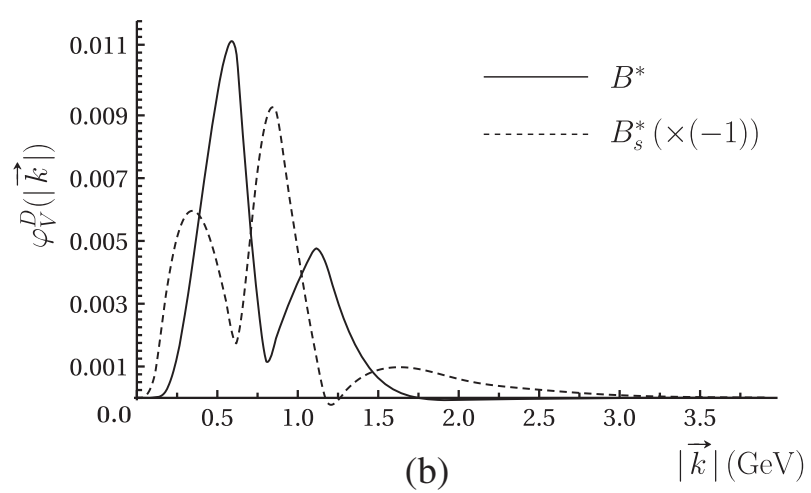

(b) mesons. 
TABLE II. Parameters of fitting functions for vector $B$ mesons, S-wave part.

\begin{tabular}{lcrrr}
\hline \hline Mesons & $a_{1}\left(\mathrm{GeV}^{-3 / 2}\right)$ & $a_{2}\left(\mathrm{GeV}^{-2}\right)$ & $a_{3}\left(\mathrm{GeV}^{-1}\right)$ & \multicolumn{1}{c}{$a_{4}$} \\
\hline$B^{*}$ & $1.62_{-0.08}^{+0.06}$ & $-1.09_{-0.11}^{+0.14}$ & $-1.23_{-0.09}^{+0.13}$ & $0.03 \pm 0.01$ \\
$B_{s}^{*}$ & $1.45_{-0.06}^{+0.04}$ & $-1.17_{-0.11}^{+0.15}$ & $-0.84_{-0.10}^{+0.09}$ & $-0.03 \pm 0.01$ \\
\hline \hline
\end{tabular}

radial wave function, and we use $\psi_{V}^{D}(\vec{k})$ for the D-wave function.

The radial wave functions are shown in Fig. 2. The contribution of the D-wave part to the leptonic decay is rather small. Therefore, the fitting functions are provided only for the S-wave part, using the same analytic form in Eq. (6). The parameters for vector mesons are collected in Table II.

(3) For higher excited states $1^{+}$and $2^{+}$, only a few states have been found in experiments up to now, and information about their decay channels is very limited. Therefore, we only show the radial wave functions for $J^{P}=1^{+}$mesons in Fig. 3 and those for $J^{P}=2^{+}$mesons in Fig. 4, without giving an analytic form. In principle, the numerical form for the wave functions can be used directly to calculate the meson decays.

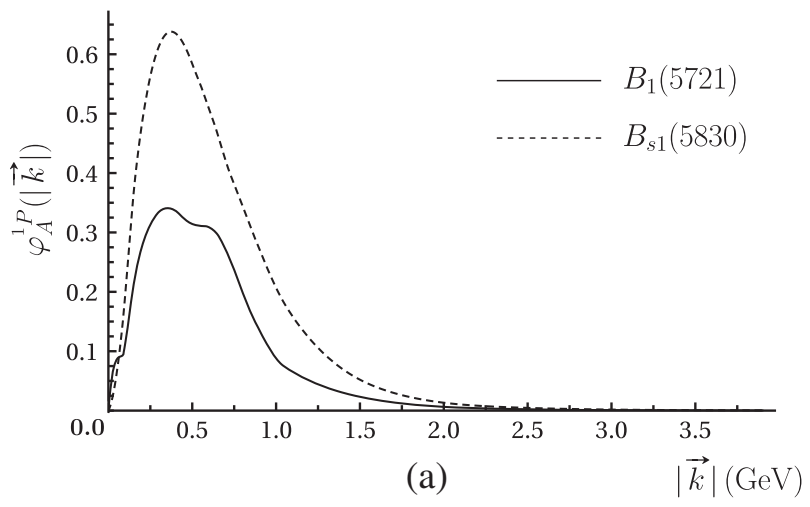

\section{B. Decay constants}

Using the wave function of the quark-antiquark bound state, the state of a bottom meson can be written as $[7,13]$

$$
\begin{aligned}
|M(\vec{P})\rangle= & \frac{1}{\sqrt{3 N_{L}}} \sum_{i} \int d^{3} k_{q} d^{3} k_{Q} \delta^{(3)}\left(\vec{P}-\vec{k}_{q}-\vec{k}_{Q}\right) \\
& \times \psi_{n l m}\left(\vec{k}_{q}\right) X_{S m_{s}}\left(a_{\vec{k}_{Q}, s_{1}}^{i \dagger}, b_{\vec{k}_{q}, s_{2}}^{i \dagger}\right)|0\rangle,
\end{aligned}
$$

where $X_{S m_{s}}$ is the spin wave function, $a_{\vec{k}_{Q}, s_{1}}^{i \dagger}, b_{\vec{k} k_{q}, s_{2}}^{i \dagger}$ are creation operators, and $S, m_{s}, s_{1}, s_{2}$ are the corresponding spin-related quantum numbers. The superscript $i$ is the color index, and the normalization factor $N_{L}$ is obtained via the normalization condition of the meson state

$$
\left\langle M(\vec{P}) \mid M\left(\overrightarrow{P^{\prime}}\right)\right\rangle=(2 \pi)^{3} 2 E \delta^{(3)}\left(\vec{P}-\vec{P}^{\prime}\right) .
$$

The anticommuting relation of the quark and antiquark annihilation and creation operators are

$$
\left\{a_{\vec{k}, s}, a_{\vec{k}^{\prime}, s^{\prime}}^{\dagger}\right\}=\delta_{s s^{\prime}} \delta^{(3)}\left(\vec{k}-\vec{k}^{\prime}\right)
$$

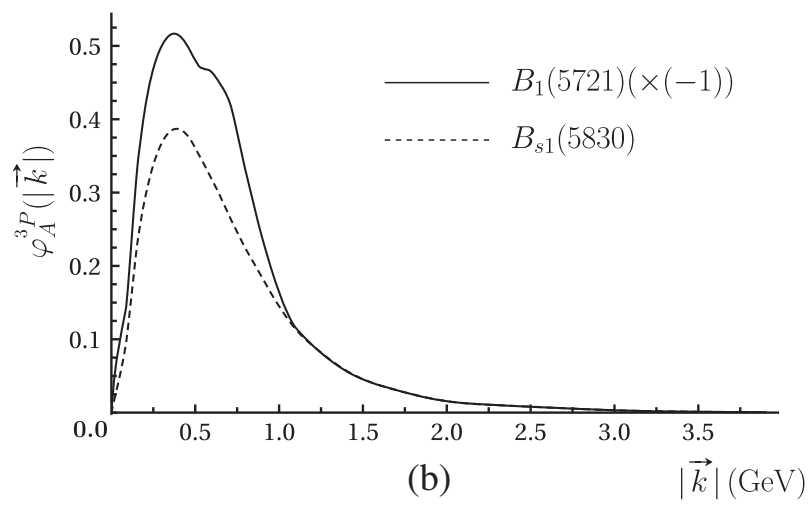

FIG. 3. Radial wave functions of axial vector $\left(1^{+}\right)$mesons (a) for the ${ }^{1} P_{1}$ parts and (b) for the ${ }^{3} P_{1}$ parts.
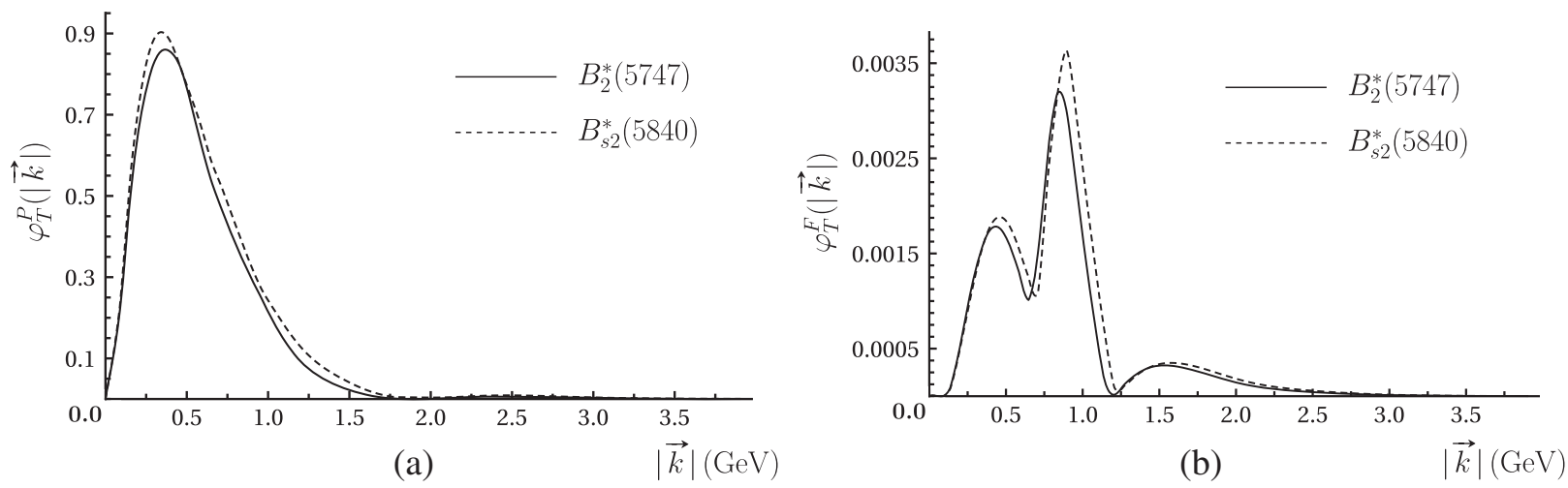

FIG. 4. Radial wave functions of tensor $\left(2^{+}\right)$mesons (a) for the P-wave parts and (b) for the F-wave parts. 


$$
\left\{b_{\vec{k}, s}, b_{\vec{k}^{\prime}, s^{\prime}}^{\dagger}\right\}=\delta_{s s^{\prime}} \delta^{(3)}\left(\vec{k}-\vec{k}^{\prime}\right)
$$

The energy and momentum conservation between the meson and its constituent quark and antiquark should hold when considering the decays of the bottom mesons. We take $k_{q}=\left(E_{q}, \vec{k}_{q}\right), k_{Q}=\left(E_{Q}, \vec{k}_{Q}\right)$, and $P=$ $\left(m_{P}, \overrightarrow{0}\right)$ as the four-momenta of the light quark, the heavy quark, and the meson in the rest frame, respectively. Due to energy and momentum conservation, one has

$$
\begin{gathered}
E_{q}+E_{Q}=m_{P}, \\
\vec{k}_{q}=-\vec{k}_{Q}=\vec{k} .
\end{gathered}
$$

To keep the four-momentum conservation, the heavy quark is taken off shell, while the light quark is kept on shell in the Altarelli-Cabibbo-Corbo-MaianiMartinelli (ACCMM) scenario [14,15]. Here we extend the ACCMM scenario by taking both the light and heavy quarks off shell. Using the off-shell quarks is a simple treatment for including the energy and momentum carried by the color field around the quarks. Both the masses of the light and heavy quarks are taken to be running masses,

$m_{q}(k)=\sqrt{E_{q}^{2}-|\vec{k}|^{2}}, \quad m_{Q}(k)=\sqrt{E_{Q}^{2}-|\vec{k}|^{2}}$.
The running masses $m_{q}(k)$ and $m_{Q}(k)$ are restricted to be positive in this work. With Eqs. (12) and (13), one can obtain

$$
\sqrt{m_{q}^{2}(k)+|\vec{k}|^{2}}+\sqrt{m_{Q}^{2}(k)+|\vec{k}|^{2}}=m_{P} .
$$

It is not enough to determine the explicit dependence of the running masses $m_{q, Q}(k)$ on the quark momentum $k$ with the above equation. We assume the ratio of $m_{q}(k) / m_{Q}(k)$ is a fixed parameter in this work; i.e., we define the ratio for each quark-antiquark pair as

$$
R_{i} \equiv \frac{m_{q}(\vec{k})}{m_{Q}(\vec{k})}, \quad i=(\bar{u} b) \sim(\bar{d} b),(\bar{s} b),(\bar{c} b) .
$$

In our numerical treatment in the following, we find that the fixed ratio $R_{i}$ can indeed accommodate the experimental data for the measured leptonic decays of the bottom mesons, and the value of the ratio $R_{i}$ is approximately around the ratio of current masses of the light and heavy quarks $m_{q} / m_{b}$.

In general, decay constants of pseudoscalar mesons $\left(B^{ \pm}, B_{d}, B_{s}, B_{c}^{ \pm}\right)$are defined as

$$
\left\langle 0\left|\bar{q} \gamma_{\mu} \gamma^{5} b\right| B(P)\right\rangle=i f_{P} P_{\mu} .
$$

Substituting Eq. (7) into the Eq. (16), we obtain the decay constant

$$
f_{P}=\sqrt{\frac{3}{(2 \pi)^{3} m_{P}}} \int d^{3} k \psi_{100}(\vec{k})\left(\sqrt{1+\frac{m_{q}(k)}{E_{q}}} \sqrt{1+\frac{m_{Q}(k)}{E_{Q}}}-\sqrt{1-\frac{m_{q}(k)}{E_{q}}} \sqrt{1-\frac{m_{Q}(k)}{E_{Q}}}\right) .
$$

For vector mesons $B^{* \pm}, B^{*}, B_{s}^{*}$, there are two types of decay constants that are defined according to different currents:

$$
\left\langle 0\left|\bar{q} \gamma_{\mu} b\right| B^{*}(P, \epsilon)\right\rangle=m_{V^{*}} f_{V^{*}} \epsilon_{\mu}, \quad\left\langle 0\left|\bar{q} \sigma_{\mu \nu} b\right| B^{*}(P, \epsilon)\right\rangle=-i f_{V^{*}}^{T}\left(P_{\mu} \epsilon_{\nu}-\epsilon_{\mu} P_{\nu}\right),
$$

where $\epsilon_{\mu}$ is the polarization vector and $\sigma_{\mu \nu}=\frac{i}{2}\left[\gamma_{\mu}, \gamma_{\nu}\right]$ is the Dirac tensor matrix. Similarly, the decay constants can be obtained as

$$
\begin{gathered}
f_{V^{*}}=\sqrt{\frac{3}{(2 \pi)^{3} m_{V^{*}}}} \int d^{3} k \psi_{n 00}^{\prime}(\vec{k})\left(\sqrt{1+\frac{m_{q}(k)}{E_{q}}} \sqrt{1+\frac{m_{Q}(k)}{E_{Q}}}+\frac{1}{3} \sqrt{1-\frac{m_{q}(k)}{E_{q}}} \sqrt{1-\frac{m_{Q}(k)}{E_{Q}}}\right), \\
f_{V^{*}}^{T}=\sqrt{\frac{3}{(2 \pi)^{3} m_{V^{*}}}} \int d^{3} k \psi_{n 00}^{\prime}(\vec{k})\left(\sqrt{1+\frac{m_{q}(k)}{E_{q}}} \sqrt{1+\frac{m_{Q}(k)}{E_{Q}}}-\frac{1}{3} \sqrt{1-\frac{m_{q}(k)}{E_{q}}} \sqrt{1-\frac{m_{Q}(k)}{E_{Q}}}\right) .
\end{gathered}
$$

The vector mesons are mixing states of $\left|n_{1}{ }^{3} S_{1}\right\rangle$ and $\left|n_{2}{ }^{3} D_{1}\right\rangle$ with $n_{1}, n_{2}=1,2,3$ (details can be found in Refs. [8,9]). The wave function $\psi_{n 00}^{\prime}(\vec{k})$ in Eqs. (19a) and (19b) is the sum of all the S-wave states. The D-wave states do not contribute to the decay constants. 


\section{Branching ratios of purely leptonic decays}

We calculate the purely leptonic decays of pseudoscalar and vector $b$-flavored mesons in this section. The decay modes we consider in this work include $B_{u(c)}^{ \pm} \rightarrow l^{ \pm} \nu_{l}$,
$B_{u(c)}^{* \pm} \rightarrow l^{ \pm} \nu_{l}, \quad B_{d(s)}^{0} \rightarrow l^{+} l^{-}$, and $B_{d(s)}^{* 0} \rightarrow l^{+} l^{-}$. For the leptonic decays of charged bottom mesons, the decay amplitudes are dominated by the tree-level diagrams. The branching ratios are calculated to be

$$
\begin{gathered}
\mathcal{B}\left(B_{q}^{ \pm} \rightarrow l^{ \pm} \nu_{l}\right)=\frac{G_{F}^{2} m_{l}^{2} M_{B_{q}}}{8 \pi}\left(1-\frac{m_{l}^{2}}{M_{B_{q}}^{2}}\right)^{2} f_{B_{q}}^{2}\left|V_{q b}\right|^{2} \tau_{B_{q}}, \\
\mathcal{B}\left(B_{q}^{* \pm} \rightarrow l^{ \pm} \nu_{l}\right)=\frac{G_{F}^{2} M_{B_{q}^{*}}^{3}\left(1-\frac{3}{2} \frac{m_{l}^{2}}{M_{B_{q}^{*}}^{2}}+\frac{1}{2} \frac{m_{l}^{6}}{M_{B_{q}^{*}}^{6}}\right) \times f_{B_{q}^{*}}^{2}\left|V_{q b}\right|^{2} \tau_{B_{q}^{*}},}{}
\end{gathered}
$$

where $G_{F}$ is the Fermi constant, $V_{q b}$ is the CKM matrix element, and $M_{B_{q}^{(*)}}$ and $m_{l}$ are the masses of the $B_{q}^{(*) \pm}$ meson and lepton, respectively. $\tau_{B_{q}^{(*)}}$ is the lifetime of the bottom meson.

For the leptonic decays of the neutral bottom mesons, the decays are induced by penguin diagrams. The effective Hamiltonian that describes such decays is [16-18]

$$
\mathcal{H}_{\mathrm{eff}}=-\frac{G_{F}}{\sqrt{2}} \lambda_{q} \sum_{i=1}^{10} C_{i}(\mu) Q_{i}(\mu)
$$

where $\lambda_{q}=V_{t b} V_{t q}^{*}$, and the operators are (we use $b \rightarrow s$ as an example and $b \rightarrow d$ is similar)

$$
\begin{aligned}
Q_{1}=\left(\bar{s}_{\alpha} c_{\beta}\right)_{V-A}\left(\bar{c}_{\beta} b_{\alpha}\right)_{V-A}, & Q_{2}=(\bar{s} c)_{V-A}(\bar{c} b)_{V-A}, \\
Q_{3}=(\bar{s} b)_{V-A} \sum_{q}(\bar{q} q)_{V-A}, & Q_{4}=\left(\bar{s}_{\alpha} b_{\beta}\right)_{V-A} \sum_{q}\left(\bar{q}_{\beta} q_{\alpha}\right)_{V-A}, \\
Q_{5} & =(\bar{s} b)_{V-A} \sum_{q}(\bar{q} q)_{V+A}, \quad Q_{6}=\left(\bar{s}_{\alpha} b_{\beta}\right)_{V-A} \sum_{q}\left(\bar{q}_{\beta} q_{\alpha}\right)_{V+A}, \\
Q_{7} & =\frac{\alpha_{e m}}{2 \pi} m_{b} \bar{s}_{\alpha} \sigma^{\mu \nu}\left(1+\gamma^{5}\right) b_{\alpha} F_{\mu \nu}, \quad Q_{8}=\frac{\alpha_{s}}{2 \pi} m_{b} \bar{s}_{\alpha} \sigma^{\mu \nu}\left(1+\gamma^{5}\right) T_{\alpha \beta}^{a} b_{\beta} G_{\mu \nu}^{a}, \\
Q_{9} & =\frac{\alpha}{2 \pi}(\bar{s} b)_{V-A}(\bar{l} l)_{V}, \quad Q_{10}=\frac{\alpha}{2 \pi}(\bar{s} b)_{V-A}(\bar{l} l)_{A},
\end{aligned}
$$

where $\alpha=\frac{e^{2}}{4 \pi}$ is the electromagnetic coupling constant. Except for the contributions of the operators $Q_{7}$ and $Q_{9}$, the operators $Q_{1-6,8}$ also contribute to the decay process $B_{d(s)}^{* 0} \rightarrow l^{+} l^{-}$up to next-to-leading order (NLO) in $\alpha_{s}$ expansion in QCD. The contributions from the operators $Q_{1-6,8}$ can be absorbed by a redefinition of two effective Wilson coefficients $C_{7,9} \rightarrow C_{7,9}^{\text {eff }}$. The explicit form of $C_{7,9}^{\text {eff }}$ can be found in Refs. [18-20]; we do not repeat it here.

Next, we give the branching ratio of the pure leptonic decay of the neutral bottom mesons. The branching ratio of $B_{d(s)}^{0} \rightarrow l^{+} l^{-}$is [21]

$\overline{\mathcal{B}}\left(B_{q} \rightarrow l^{+} l^{-}\right)=\frac{G_{F}^{2} M_{B_{q}} m_{l}^{2} \alpha^{2}}{4 \pi^{3} \Gamma_{H}^{q}} f_{B_{q}}^{2}\left|\lambda_{q}\right|^{2} \sqrt{1-\frac{4 m_{l}^{2}}{M_{B_{q}}^{2}}}\left|C_{10}\right|^{2}$.

The bar over $\mathcal{B}$ indicates that it is the averaged timeintegrated branching ratio that depends on the details of
$B_{q}^{0}-\bar{B}_{q}^{0}$ mixing [22]. $\Gamma_{H}^{q}$ denotes the total decay width of the heavier mass eigenstate. In Ref. [21], the authors define a different Wilson coefficient $C_{A}$, and its relation to $C_{10}$ in Eq. (21) is straightforward: $\left|C_{A}\right|=\frac{\sin ^{2} \theta_{W}}{2}\left|C_{10}\right|$, where $\theta_{W}$ is the weak-mixing angle (the Weinberg angle).

For the process of the vector meson decaying into a charged lepton-antilepton pair, the branching ratio reads [23]

$$
\begin{aligned}
\overline{\mathcal{B}}\left(B_{q}^{*} \rightarrow l^{+} l^{-}\right)= & \frac{1}{\Gamma_{B_{q}^{*}}} \frac{G_{F}^{2} M_{B_{q}^{*}}^{3} \alpha^{2}}{96 \pi^{3}} f_{B_{q}^{*}}^{2}\left|\lambda_{q}\right|^{2} \\
& \times\left(\left|C_{9}^{\mathrm{eff}}+2 \frac{m_{b} f_{B_{q}^{*}}^{T}}{M_{B_{q}^{*}} f_{B_{q}^{*}}^{*}} C_{7}^{\text {eff }}\right|^{2}+\left|C_{10}\right|^{2}\right),
\end{aligned}
$$

where the contributions of order $\mathcal{O}\left(m_{l}^{2} / M_{B_{q}^{*}}^{2}\right)$ are neglected and $m_{c} \ll m_{b}$ is considered. 
TABLE III. Numerical inputs.

\begin{tabular}{|c|c|c|c|c|c|}
\hline Parameter & Value & Unit & Parameter & Value & Unit \\
\hline$\overline{G_{F}}$ & $1.16638 \times 10^{-5}$ & $\mathrm{GeV}^{-2}$ & $\left|V_{u b}\right|$ & $3.94(36) \times 10^{-3}$ & $\cdots$ \\
\hline$\alpha_{s}^{(5)}\left(M_{z}\right)$ & $0.1181(11)$ & $\cdots$ & $\left|V_{c b}\right|$ & $4.22(8) \times 10^{-2}$ & $\cdots$ \\
\hline$\alpha^{(5)}\left(M_{Z}\right)$ & $1 / 127.955(10)$ & $\cdots$ & $\left|V_{t d}\right|$ & $8.1(5) \times 10^{-3}$ & $\cdots$ \\
\hline$M_{Z}$ & $91.1876(21)$ & $\mathrm{GeV}$ & $\left|V_{t s}\right|$ & $3.94(23) \times 10^{-2}$ & $\cdots$ \\
\hline$M_{t}$ & $173.1(9)$ & $\mathrm{GeV}$ & $\left|V_{t b}\right|$ & $1.019(25)$ & $\ldots$ \\
\hline$M_{B^{ \pm}}$ & $5279.32(14)$ & $\mathrm{MeV}$ & $M_{B^{*}}$ & $5324.65(25)$ & $\mathrm{MeV}$ \\
\hline$M_{B_{s}}$ & $5366.89(19)$ & $\mathrm{MeV}$ & $M_{B_{s}^{*}}$ & $5415.4_{-15}^{+1.8}$ & $\mathrm{MeV}$ \\
\hline$M_{B_{d}}$ & $5279.63(15)$ & $\mathrm{MeV}$ & $M_{B_{c}}$ & $6274.9(8)$ & $\mathrm{MeV}$ \\
\hline$\tau_{B^{ \pm}}$ & $1.638(4)$ & ps & $\tau_{B_{d}}$ & $1.520(4)$ & ps \\
\hline $2 /\left(\Gamma_{B_{s L}}+\Gamma_{B_{s H}}\right)$ & $1.509(4)$ & ps & $\Gamma_{B_{s L}}-\Gamma_{B_{s H}}$ & $0.088(6)$ & $\mathrm{ps}^{-1}$ \\
\hline
\end{tabular}

\section{NUMERICAL CALCULATION}

In this section, we calculate the decay constants in Eqs. (17), (19) and the leptonic decay branching ratios in Eqs. (20), (22), (23) numerically, and compare them with experimental measurements.

The parameters used in this work, which are collected in Table III, are quoted from PDG [24].

(1) For pseudoscalar $B$ mesons $\left(J^{P}=0^{-}\right)$:

The $J^{P}=0^{-}$pseudoscalar meson is the $\left|1^{1} S_{0}\right\rangle$ state. Besides the parameters in Table III, additional numerical input is the Wilson coefficient $C_{10}$, whose expression is known up to next-to-leading order in electroweak (EW) corrections [25] and next-to-next-to-leading order (NNLO) in QCD [26]. Its value is $\left|C_{10}\left(\mu_{b}=m_{b} \sim 5 \mathrm{GeV}\right)\right|=$ $4.053 \pm 0.032$.

Our numerical results are listed in Table IV. The uncertainties of branching ratios mainly come from decay constants (whose relative error is $5 \%$ in our scenario) and CKM matrix elements (see the full review in Ref. [24]). For the two well-measured decay modes $B^{+} \rightarrow \tau^{+} \nu_{\tau}$ and $B_{s} \rightarrow \mu^{+} \mu^{-}$, our results are in good agreement with experimental data. The other predicted branching ratios are all consistent with experimental data. They are well below the experimental upper limit. The branching ratios of $B^{+} \rightarrow \mu^{+} \nu_{\mu}$ and $B_{d} \rightarrow \mu^{+} \mu^{-}$are very near to the present experimental upper limit, and there is hope they may be detected with the upgraded detectors at Belle II and/or $\mathrm{LHCb}$ [27] in the near future.

In addition, the prediction of the branching ratio of $B_{c}^{+} \rightarrow \tau^{+} \nu_{\tau}$ is 2 orders of magnitude larger than that of $B^{+}$. Therefore, it could be a possible channel to be measured in experiments in the future.

(2) For vector $B$ mesons $\left(J^{P}=1^{-}\right)$:

Since the total decay widths of vector $B$ mesons are not well measured in experiments up to now, in this work, their values of theoretical estimations will be used. As stated in Refs. [33-35], vector $B$ mesons' decays are dominated by the electromagnetic processes $B^{*} \rightarrow B \gamma$, and thus we make the assumption that the total decay width $\Gamma$ approximately equals $\Gamma\left(B^{*} \rightarrow B \gamma\right)$. The respective values are $\Gamma_{B_{s}^{*}} \sim 0.068(18) \mathrm{keV}, \Gamma_{B_{d}^{*}} \sim$ $0.148(20) \mathrm{keV}$, and $\Gamma_{B^{*+}} \sim 0.468_{-0.075}^{+0.073} \mathrm{keV}$. The "running" mass of the $b$ quark is $m_{b}(\overline{\mathrm{MS}})=4.18 \mathrm{GeV}$. The values of the effective Wilson coefficients are

TABLE IV. Decay constants, purely leptonic branching ratios of pseudoscalars.

\begin{tabular}{|c|c|c|c|c|c|}
\hline$B$ meson & $R_{i}[$ Eq. (15)] & $f_{B}(\mathrm{MeV})$ & Channel & This work & Experiment \\
\hline$(b q)$ & $1.0 \times 10^{-3}$ & $210(10)$ & $\begin{array}{l}B^{+} \rightarrow e^{+} \nu_{e} \\
B^{+} \rightarrow \mu^{+} \nu_{\mu} \\
B^{+} \rightarrow \tau^{+} \nu_{\tau} \\
B_{d} \rightarrow e^{+} e^{-} \\
B_{d} \rightarrow \mu^{+} \mu^{-} \\
B_{d} \rightarrow \tau^{+} \tau^{-}\end{array}$ & $\begin{aligned} 1.27(26) & \times 10^{-11} \\
5.4(1.1) & \times 10^{-7} \\
1.21(25) & \times 10^{-4} \\
3.00(49) & \times 10^{-15} \\
1.28(21) & \times 10^{-10} \\
2.68(44) & \times 10^{-8}\end{aligned}$ & $\begin{array}{c}<9.8 \times 10^{-7}[28] \\
<10.7 \times 10^{-7}[29] \\
1.25(28)(27) \times 10^{-4}[30] \\
<8.3 \times 10^{-8}[31] \\
<2.1 \times 10^{-10}[1] \\
<1.6 \times 10^{-3}[32]\end{array}$ \\
\hline$(b s)$ & $1.0 \times 10^{-2}$ & $229(11)$ & $\begin{array}{l}B_{s} \rightarrow e^{+} e^{-} \\
B_{s} \rightarrow \mu^{+} \mu^{-} \\
B_{s} \rightarrow \tau^{+} \tau^{-}\end{array}$ & $\begin{aligned} 8.1(1.3) & \times 10^{-14} \\
3.45(55) & \times 10^{-9} \\
7.3(1.2) & \times 10^{-7}\end{aligned}$ & $\begin{array}{l}<2.8 \times 10^{-7}[31] \\
2.8_{-0.7}^{+0.8} \times 10^{-9}[1] \\
<5.2 \times 10^{-3}[32]\end{array}$ \\
\hline$(b c)$ & 0.3 & $429(21)$ & $\begin{array}{l}B_{c}^{+} \rightarrow e^{+} \nu_{e} \\
B_{c}^{+} \rightarrow \mu^{+} \nu_{\mu} \\
B_{c}^{+} \rightarrow \tau^{+} \nu_{\tau}\end{array}$ & $\begin{array}{r}2.24(24) \times 10^{-9} \\
9.6(1.0) \times 10^{-5} \\
2.29(24) \times 10^{-2}\end{array}$ & $\begin{array}{l}\cdots \\
\cdots \\
\cdots\end{array}$ \\
\hline
\end{tabular}


TABLE V. Decay constants, purely leptonic branching ratios of vectors with $l=e, \mu$.

\begin{tabular}{lccccc}
\hline \hline$B$ meson & $R_{i}$ [Eq. (15)] & $f_{B_{q}^{*}}(\mathrm{MeV})$ & $f_{B_{q}^{*}}^{T}(\mathrm{MeV})$ & Channel & This work \\
\hline$(b q)$ & $1.0 \times 10^{-3}$ & $223(16)$ & $201(14)$ & $B^{*+} \rightarrow e^{+} \nu_{e}$ & $9.0(2.5) \times 10^{-10}$ \\
& & & $B^{*+} \rightarrow \mu^{+} \nu_{\mu}$ & $9.0(2.5) \times 10^{-10}$ \\
& & & $B^{*+} \rightarrow \tau^{+} \nu_{\tau}$ & $7.5(2.1) \times 10^{-10}$ \\
& & & $B_{d}^{*} \rightarrow l^{+} l^{-}$ & $3.16(77) \times 10^{-13}$ \\
$(b s)$ & $1.0 \times 10^{-2}$ & $242(17)$ & $219(15)$ & $B_{s}^{*} \rightarrow l^{+} l^{-}$ & $2.02(64) \times 10^{-11}$ \\
\hline \hline
\end{tabular}

$C_{9}^{\mathrm{eff}}=C_{9}^{\mathrm{eff}}\left(m_{b}, m_{B^{*}}^{2}\right) \sim C_{9}^{\mathrm{eff}}\left(m_{b}, m_{B_{s}^{*}}^{2}\right)=4.560+i 0.612$ and $\quad C_{7}^{\text {eff }}=C_{7}^{\text {eff }}\left(m_{b}, m_{B^{*}}^{2}\right) \sim C_{7}^{\text {eff }}\left(m_{b}, m_{B_{s}^{*}}^{2}\right)=-0.384-$ $i 0.111$ [23].

The results for the branching ratios of vector $B$ mesons are shown in Table V. The uncertainties mainly come from the uncertainties of the decay width of vector mesons and CKM parameters. In general, the branching ratios of the leptonic decay of vector $B$ mesons are very small.

\section{SUMMARY}

To summarize, we study the leptonic decays of $b$-flavored mesons in the relativistic potential model. The decay constants of the bottom mesons and the branching ratios of the leptonic decay modes are calculated.
The predictions for the branching ratios of $B^{+} \rightarrow \tau^{+} \nu_{\tau}$ and $B_{s} \rightarrow \mu^{+} \mu^{-}$are well consistent with the experimental measurements. The other predicted branching ratios are well below the experimental upper limit. For $B^{+} \rightarrow \mu^{+} \nu_{\mu}$ and $B_{d} \rightarrow \mu^{+} \mu^{-}$decays, the predictions of the branching ratios are very near to the present experimental upper limit, which are very hopeful to be detected with the upgraded detectors at Belle II and/or LHCb in the near future. For the leptonic decays of the vector $B$ mesons, the branching ratios are very small.

\section{ACKNOWLEDGMENTS}

This work is supported in part by the National Natural Science Foundation of China under Contracts No. 11875168 and No. 11375088.
[1] M. Aaboud et al. (ATLAS Collaboration), Study of the rare decays of $B_{s}^{0}$ and $B^{0}$ mesons into muon pairs using data collected during 2015 and 2016 with the ATLAS detector, arXiv:1812.03017.

[2] A. Crivellin et al., PSI/UZH Workshop: Impact of $B \rightarrow$ $\mu^{+} \mu^{-}$on new physics searches, arXiv:1803.10097.

[3] R. Fleischer, R. Jaarsma, and G. Tetlalmatzi-Xolocotzi, In pursuit of new physics with $B_{s, d}{ }^{0} \rightarrow \ell^{+} \ell^{-}, \mathrm{J}$. High Energy Phys. 05 (2017) 156.

[4] N. Cabibbo, Unitary Symmetry and Leptonic Decays, Phys. Rev. Lett. 10, 531 (1963).

[5] M. Kobayashi and T. Maskawa, CP-Violation in the renormalizable theory of weak interaction, Prog. Theor. Phys. 49, 652 (1973).

[6] H.-K. Sun and M.-Z. Yang, Decay constants and distribution amplitudes of B meson in the relativistic potential model, Phys. Rev. D 95, 113001 (2017).

[7] M.-Z. Yang, Wave functions and decay constants of B and D mesons in the relativistic potential model, Eur. Phys. J. C 72, 1880 (2012).

[8] J.-B. Liu and M.-Z. Yang, Spectrum of the charmed and b-flavored mesons in the relativistic potential model, J. High Energy Phys. 07 (2014) 106.

[9] J.-B. Liu and M.-Z. Yang, Spectrum of higher excitations of $\mathrm{B}$ and $\mathrm{D}$ mesons in the relativistic potential model, Phys. Rev. D 91, 094004 (2015).
[10] S. Godfrey and N. Isgur, Mesons in a relativized quark model with chromodynamics, Phys. Rev. D 32, 189 (1985).

[11] E. Eichten, K. Gottfried, T. Kinoshita, K. D. Lane, and T.-M. Yan, Charmonium: The model, Phys. Rev. D 17, 3090 (1978); Erratum 21, 313 (1980).

[12] E. Eichten, K. Gottfried, T. Kinoshita, K. D. Lane, and T.-M. Yan, Charmonium: Comparison with experiment, Phys. Rev. D 21, 203 (1980).

[13] H. Leutwyler and M. Roos, Determination of the elements $V_{\text {us }}$ and $V_{\text {ud }}$ of the Kobayashi-Maskawa matrix, Z. Phys. C 25, 91 (1984).

[14] G. Altarelli, N. Cabibbo, G. Corbo, L. Maiani, and G. Martinelli, Leptonic decay of heavy flavors: A theoretical update, Nucl. Phys. B208, 365 (1982).

[15] P. Colangelo, F. De Fazio, M. Ladisa, G. Nardulli, P. Santorelli, and A. Tricarico, Semileptonic and rare B-meson transitions in a QCD relativistic potential model, Eur. Phys. J. C8, 81 (1999).

[16] B. Grinstein, M. J. Savage, and M. B. Wise, $B \rightarrow X(s) e^{+} e^{-}$ in the six quark model, Nucl. Phys. B319, 271 (1989).

[17] B. Grinstein, R. P. Springer, and M. B. Wise, Strong interaction effects in weak radiative $\bar{B}$ meson decay, Nucl. Phys. B339, 269 (1990).

[18] B. Grinstein and D. Pirjol, Exclusive rare $B \rightarrow K^{*} \ell^{+} \ell^{-}$ decays at low recoil: Controlling the long-distance effects, Phys. Rev. D 70, 114005 (2004). 
[19] D. Seidel, Analytic two-loop virtual corrections to $b \rightarrow d l^{+} l^{-}$, Phys. Rev. D 70, 094038 (2004).

[20] C. Greub, V. Pilipp, and C. Schupbach, Analytic calculation of two-loop QCD corrections to $b \rightarrow s l^{+} l^{-}$in the high $q^{2}$ region, J. High Energy Phys. 12 (2008) 040.

[21] C. Bobeth, M. Gorbahn, T. Hermann, M. Misiak, E. Stamou, and M. Steinhauser, $B_{s, d} \rightarrow l^{+} l^{-}$in the Standard Model with Reduced Theoretical Uncertainty, Phys. Rev. Lett. 112, 101801 (2014).

[22] K. De Bruyn, R. Fleischer, R. Knegjens, P. Koppenburg, M. Merk, A. Pellegrino, and N. Tuning, Probing New Physics via the $B_{s}^{0} \rightarrow \mu^{+} \mu^{-}$Effective Lifetime, Phys. Rev. Lett. 109, 041801 (2012).

[23] B. Grinstein and J. Martin Camalich, Weak Decays of Excited B Mesons, Phys. Rev. Lett. 116, 141801 (2016).

[24] M. Tanabashi et al. (Particle Data Group), Review of particle physics, Phys. Rev. D 98, 030001 (2018).

[25] C. Bobeth, M. Gorbahn, and E. Stamou, Electroweak corrections to $B_{s, d} \rightarrow \ell^{+} \ell^{-}$, Phys. Rev. D 89, 034023 (2014).

[26] T. Hermann, M. Misiak, and M. Steinhauser, Three-loop QCD corrections to $B_{s} \rightarrow \mu^{+} \mu^{-}$, J. High Energy Phys. 12 (2013) 097.

[27] J. Albrecht, F. Bernlochner, M. Kenzie, S. Reichert, D. Straub, and A. Tully, Future prospects for exploring present day anomalies in flavour physics measurements with Belle II and LHCb, arXiv:1709.10308.
[28] N. Satoyama et al. (Belle Collaboration), A search for the rare leptonic decays $B^{+} \rightarrow \mu^{+} \nu_{\mu}$ and $B^{+} \rightarrow e^{+} \nu_{e}$, Phys. Lett. B 647, 67 (2007).

[29] A. Sibidanov et al. (Belle Collaboration), Search for $B^{-} \rightarrow$ $\mu^{-} \bar{\nu}_{\mu}$ Decays at the Belle Experiment, Phys. Rev. Lett. 121, 031801 (2018).

[30] B. Kronenbitter et al. (Belle Collaboration), Measurement of the branching fraction of $B^{+} \rightarrow \tau^{+} \nu_{\tau}$ decays with the semileptonic tagging method, Phys. Rev. D 92, 051102 (2015).

[31] T. Aaltonen et al. (CDF Collaboration), Search for the Decays $B_{(s)}^{0} \rightarrow e^{+} \mu^{-}$and $B_{(s)}^{0} \rightarrow e^{+} e^{-}$in CDF Run II, Phys. Rev. Lett. 102, 201801 (2009).

[32] R. Aaij et al. (LHCb Collaboration), Search for the decays $B_{s}^{0} \rightarrow \tau^{+} \tau^{-}$and $B^{0} \rightarrow \tau^{+} \tau^{-}$, Phys. Rev. Lett. 118, 251802 (2017).

[33] Q. Chang, J. Zhu, N. Wang, and R.-M. Wang, Probing the effects of new physics in $\bar{B}^{*} \rightarrow P \ell \bar{\nu}_{\ell}$ decays, Adv. High Energy Phys. 2018, 7231354 (2018).

[34] H.-M. Choi, Decay constants and radiative decays of heavy mesons in light-front quark model, Phys. Rev. D 75, 073016 (2007).

[35] C.-Y. Cheung and C.-W. Hwang, Strong and radiative decays of heavy mesons in a covariant model, J. High Energy Phys. 04 (2014) 177. 